\title{
THREE-DIMENSIONAL ALMOST $\alpha$-PARA-KENMOTSU MANIFOLDS SATISFYING CERTAIN NULLITY CONDITIONS *
}

\author{
Ximin Liu and Quanxiang Pan
}

\begin{abstract}
In this paper, we study 3-dimensional almost $\alpha$-para-Kenmotsu manifolds satisfying special types of nullity conditions depending on smooth functions $\tilde{\kappa}, \tilde{\mu}$ and $\tilde{\nu}=$ constant, also we present a local description of the structure of a 3-dimensional almost $\alpha$-para-Kenmotsu $(\tilde{\kappa}, \tilde{\mu}, \tilde{\nu}=$ const. $)$-manifold $(M, \tilde{\varphi}, \xi, \eta, \tilde{g})$ with $\tilde{\kappa}+\alpha^{2} \neq 0$ such that $d \tilde{\kappa} \wedge \eta=0$.
\end{abstract}

Keywords: Almost paracontact metric manifold; almost $\alpha$-para-Kenmotsu manifold; nullity distribution.

\section{Introduction}

The aim of this paper is to study the local description of almost $\alpha$-para-Kenmotsu manifolds. Kenmotsu manifolds have been introduced and studied by K. Kenmotsu in 1972 [10], and the geometry of almost Kenmotsu manifolds have been investigated in many aspects [5]-[7]. Most of the results contained in [5]-[6] can be easily generalized to the class of almost $\alpha$-Kenmotsu manifolds, where $\alpha$ is a non-zero real number [7]. Many authors have investigated the geometry of contact metric manifolds whose characteristic vector field $\xi$ belongs to the $(\kappa, \mu)$-nullity distribution, i.e. the curvature tensor field satisfies the condition

$$
R(X, Y) \xi=\kappa(\eta(Y) X-\eta(X) Y)+\mu(\eta(Y) h X-\eta(X) h Y),
$$

for some real numbers $\kappa$ and $\mu$, where $2 h$ denotes the Lie derivative of $\varphi$ in the direction of $\xi$. This new class of Riemannian manifolds was introduced in [4] as a natural generalization both of the Sasakian condition $R(X, Y) \xi=\eta(Y) X-\eta(X) Y$ and of those contact metric manifolds satisfying $R(X, Y) \xi=0$ which were studied by D.E. Blair in [3]. Koufogiorgos and Tsichlias found a new class of 3-dimensional contact metric manifolds that $\kappa$ and $\mu$ are non-constant smooth functions[11]. They generalized $(\kappa, \mu)$-contact metric manifolds for dimensions greater than three on

Received November 29, 2016; accepted February 03, 2017

2010 Mathematics Subject Classification. Primary 53C25; Secondary 53D10

* The authors were supported in part by NSFC (No. 11371076 and 11401178) 
non-Sasakian manifolds, where the functions $\kappa, \mu$ are constant. Nowadays contact metric $(\kappa, \mu)$-space is considered as a very important topic in contact Riemannian geometry. Following these works, P. Dacko and Z. Olszak studied almost cosymplectic $(\kappa, \mu, \nu)$-spaces in [12], whose almost cosymplectic structures $(\varphi, \xi, \eta, g)$ satisfy the condition

$$
R(X, Y) \xi=\eta(Y)(\kappa I+\mu h+\nu \varphi h) X-\eta(X)(\kappa I+\mu h+\nu \varphi h) Y,
$$

for $\kappa, \mu, \nu \in R_{\eta}\left(M^{2 n+1}\right)$, where $R_{\eta}\left(M^{2 n+1}\right)$ is the ring of smooth functions $f$ on $M^{2 n+1}$ for which $d f \wedge \eta=0$. Later, [8] studied the generalized almost cosymplectic $(\kappa, \mu, \nu)$-spaces, that is: almost $\alpha$-cosymplectic $(\kappa, \mu, \nu)$-spaces and also pointed out that the nullity condition is invariant under $D$-homothetic deformation of almost cosymplectic $(\kappa, \mu, \nu)$-spaces in all dimensions.

The study of paracontact geometry was initiated by S. Kaneyuki and F.L. Williams in [14] and then it was continued by many other authors in many aspects, for example, a systematic study of paracontact metric manifolds, and some remarkable subclasses like para-Sasakian manifolds, was carried out by S. Zamkovoy [15], a systematic study of almost $\alpha$-paracosymplectic manifolds carried by I. K. Erken, P. Dacko and C. Murathan [9], [13].The importance of paracontact geometry has been pointed out highlighting the interplays with the theory of para-Kähler manifolds and its role in pseudo-Riemannian geometry and mathematical physics. In recent years, many authors turned to the study of paracontact geometry due to an unexpected relationship between $(\kappa, \mu)$-contact metric manifold and paracontact geometry was found in [2]. It was proved that any (non-Sasakian) $(\kappa, \mu)$-contact metric manifold carries a canonical paracontact metric structure $(\tilde{\varphi}, \xi, \eta, \tilde{g})$ whose Levi-Civita connection satisfies a condition formally similar to (1.1)

$$
\tilde{R}(X, Y) \xi=\tilde{\kappa}(\eta(Y) X-\eta(X) Y)+\tilde{\mu}(\eta(Y) \tilde{h} X-\eta(X) \tilde{h} Y),
$$

where $2 \tilde{h}:=L_{\xi} \tilde{\varphi}$ and, in this case, $\tilde{\kappa}=\left(1-\frac{\mu}{2}\right)^{2}+\kappa-2, \tilde{\mu}=2$. In [1], the authors showed that while the values of $\tilde{\kappa}$ and $\tilde{\mu}$ change the form but (1.3) remains unchanged under $D$-homothetic deformations. There are differences between a $(\kappa, \mu)$-contact metric manifold $(M, \varphi, \xi, \eta, g)$ and $(\tilde{\kappa}, \tilde{\mu})$-paracontact metric manifold $(M, \tilde{\varphi}, \xi, \eta, \tilde{g})$. Namely, unlike in the contact Riemannian case, a $(\tilde{\kappa}, \tilde{\mu})$-paracontact metric manifold such that $\tilde{\kappa}=-1$ in general is not para-Sasakian. And there are $(\tilde{\kappa}, \tilde{\mu})$-paracontact metric manifold such that $\tilde{h}^{2}=0$ but with $\tilde{h} \neq 0$ in [2]. Another important difference with the contact metric manifold is that while for contact metric case $\kappa \leq 1,(\tilde{\kappa}, \tilde{\mu})$-paracontact metric manifold has no resriction for the constants $\tilde{\kappa}$ and $\tilde{\mu}$. There are similar results about almost $\alpha$-cosymplectic $\kappa, \mu, \nu$-spaces and almost $\alpha$-paracosymplectic $\kappa, \mu, \nu$-spaces [8] and [9].

Recently, in [16] V. Saltarelli studied 3-dimensional almost Kenmotsu manifolds satisfying certain nullity conditions and gave some complete local descriptions of their structure. Motivated by the unexpected relationship between almost Kenmotsu and para-Kenmotsu manifold, we study almost $\alpha$-para-Kenmotsu manifold in this paper and give a complete local description of 3-dimensional almost $\alpha$-paraKenmotsu $(\kappa, \mu, \nu)$-spaces. 
This paper is organized in the following way. In section 2, some preliminaries and properties about almost $\alpha$-para-kenmotsu manifolds are given. In section 3 , we give some results concerning almost $\alpha$-para-Kenmotsu $(\tilde{\kappa}, \tilde{\mu}, \tilde{\nu})$-spaces. In section 4 , we will give a local description of the structure of a 3-dimensional almost $\alpha$-paraKenmotsu $(\tilde{\kappa}, \tilde{\mu}, \tilde{\nu}=$ const. $)$-space with $d \tilde{\kappa} \wedge \eta=0$. We also construct in $R^{3}$ two families of such manifolds depending on $\tilde{h}$ of $\mathfrak{h}_{1}$ or $\mathfrak{h}_{3}$ type, and in the last section we give a necessary and sufficient condition for a local structure to be an almost $\alpha$ para-Kenmotsu $(\tilde{\kappa}, \tilde{\mu}, \tilde{\nu}=$ const. $)$-space with $d \tilde{\kappa} \wedge \eta=0$. All manifolds are assumed to be connected and smooth.

\section{Preliminaries}

In this section, we recall some basic facts about paracontact metric manifolds.

A $2 n+1$-dimensional smooth manifold $M$ is said to have an almost paracontact structure if it admits a $(1,1)$-tensor field $\tilde{\varphi}$, a vector field $\xi$ and a 1-form $\eta$ satisfying the following conditions:

(i) $\tilde{\varphi}^{2}=\mathrm{Id}-\eta \otimes \xi, \quad \eta(\xi)=1$,

(ii) the tensor field $\tilde{\varphi}$ induces an almost paracomplex structure on each fiber of $\mathcal{D}=\operatorname{Ker}(\eta)$, i.e. the \pm 1 -eigendistributions $\mathcal{D}^{ \pm}:=\mathcal{D}_{\tilde{\varphi}}( \pm 1)$ of $\tilde{\varphi}$ have equal dimension $n$.

From the definition it follows that $\tilde{\varphi}(\xi)=0, \eta \circ \tilde{\varphi}=0$ and $\operatorname{rank}(\tilde{\varphi})=2 n$. When the tensor field $N_{\tilde{\varphi}}:=[\tilde{\varphi}, \tilde{\varphi}]-2 d \eta \otimes \xi$ vanishes identically the almost paracontact manifold is said to be normal. If an almost paracontact manifold admits a pseudoRiemannian metric $\tilde{g}$ such that

$$
\tilde{g}(\tilde{\varphi} X, \tilde{\varphi} Y)=-\tilde{g}(X, Y)+\eta(X) \eta(Y)
$$

for any vector fields $X, Y \in \Gamma(T M)$. Then we say that $\left(M^{2 n+1}, \tilde{\varphi}, \xi, \eta, \tilde{g}\right)$ is an almost paracontact metric manifold. Notice that any such a pseudo-Riemannian metric is necessarily of signature $(n, n+1)$. For an almost paracontact metric manifold, there always exists an orthogonal basis $\left\{\xi, X_{1}, \ldots, X_{n}, Y_{1}, \ldots, Y_{n}\right\}$ such that $\tilde{g}\left(X_{i}, X_{j}\right)=\delta_{i j}, \tilde{g}\left(Y_{i}, Y_{j}\right)=-\delta_{i j}$ and $Y_{i}=\tilde{\varphi} X_{i}$, for any $i, j \in\{1, \ldots, n\}$. Such basis is called a $\varphi$-basis. The fundamental 2-form $\tilde{\Phi}$ associate with the structure is defined by $\tilde{\Phi}(X, Y)=\tilde{g}(X, \tilde{\varphi} Y)$ for all vector fields $X, Y$ on $M$. The structure is normal if the tensor field $\mathcal{N}=[\tilde{\varphi}, \tilde{\varphi}]+2 d \eta \otimes \xi$ vanishes, where $[\tilde{\varphi}, \tilde{\varphi}]$ is the Nijenhuistorsion of $\tilde{\varphi}$. For more details, we refer the reader to [15]. According to [9], an almost paracontact metric manifold $(M, \tilde{\varphi}, \xi, \eta, \tilde{g})$ is said to be an almost $\alpha$-para-Kenmotsu manifold if

$$
d \eta=0, \quad d \tilde{\Phi}=2 \alpha \eta \wedge \tilde{\Phi}, \quad \alpha=\text { const. } \neq 0 .
$$

A normal almost $\alpha$-para-Kenmotsu manifold is an $\alpha$-para-Kenmotsu manifold.

Let $(M, \tilde{\varphi}, \xi, \eta, \tilde{g})$ be an almost $\alpha$-para-Kenmotsu manifold. Since $d \eta=0$, the canonical distribution $\mathcal{D}=\operatorname{ker}(\eta)$ is completely integrable. Each leaf of the foliation, determined by $\mathcal{D}$, carries an almost para-Kähler structure $(J,<,>)$

$$
J \bar{X}=\tilde{\varphi} \bar{X}, \quad<\bar{X}, \bar{Y}>=\tilde{g}(\bar{X}, \bar{Y}),
$$


$\bar{X}, \bar{Y}$ are vector fields tangent to the leaf. If this structure is para-Kähler, leaf is called a para-Kähler leaf. Furthermore, we have $L_{\xi} \eta=0$ and $[\xi, X] \in \mathcal{D}$ for any $X \in \mathcal{D}$. Furthermore, we have $\tilde{\nabla}_{\xi} \varphi=0$, so that $\tilde{\nabla}_{\xi} \xi=0$ and $\tilde{\nabla}_{\xi} X \in \mathcal{D}$ for any $X \in \mathcal{D}$. Define $\tilde{h}=\frac{1}{2} L_{\xi} \tilde{\varphi}$, we get the following proposition,

Proposition 2.1. [9] Let $(M, \tilde{\varphi}, \xi, \eta, \tilde{g})$ be an almost $\alpha$-paracosymplectic manifold, we have the following relations:

$$
\tilde{g}(\tilde{h} X, Y)=\tilde{g}(X, \tilde{h} Y), \tilde{h} \tilde{\varphi}=-\tilde{\varphi} \tilde{h}, \tilde{h} \xi=0,
$$

$$
\begin{gathered}
\tilde{\nabla} \xi=\alpha \tilde{\varphi}^{2}+\tilde{\varphi} \tilde{h} \\
\operatorname{tr}(\tilde{h})=0, \quad \operatorname{tr}(\tilde{\varphi} \tilde{h})=0 .
\end{gathered}
$$

Moreover, also in [9], it follows that the curvature properties of an almost $\alpha$-paraKenmotsu manifold,

$$
\begin{gathered}
\tilde{R}(X, Y) \xi=\alpha \eta(X)(\alpha Y+\tilde{\varphi} \tilde{h} Y)-\alpha \eta(Y)(\alpha X+\tilde{\varphi} \tilde{h} X)+\left(\tilde{\nabla}_{X} \tilde{\varphi} \tilde{h}\right) Y-\left(\tilde{\nabla}_{Y} \tilde{\varphi} \tilde{h}\right) X \\
\left(\tilde{\nabla}_{X} \tilde{\varphi}\right) Y-\left(\tilde{\nabla}_{\tilde{\varphi} X} \tilde{\varphi}\right) \tilde{\varphi} Y=\eta(Y)(\alpha \tilde{\varphi} X-\tilde{h} X)-2 \alpha(\tilde{g}(X, \tilde{\varphi} Y) \xi+\eta(Y) \tilde{\varphi}) .
\end{gathered}
$$

Finally, we recall that an almost paracontact metric manifold $(M, \tilde{\varphi}, \xi, \eta, \tilde{g})$ is said to be $\eta$-Einstein if its Ricci tensor satisfies

$$
\tilde{R i c}=a \tilde{g}+b \eta \oplus \eta
$$

or equivalently

$$
\tilde{Q}=a I+b \eta \otimes \xi
$$

where $a$ and $b$ are smooth functions on $M^{2 n+1}$. A vector field $X \in T_{p} M$ is called Killing vector field if $\mathcal{L}_{X} \tilde{g}=0$, that is, $\tilde{g}\left(\tilde{\nabla}_{Y} X, Z\right)+\tilde{g}\left(\tilde{\nabla}_{Z} X, Y\right)=0$, where $Y, Z \in$ $T_{p} M$ are arbitrary vector fields.

In [9], Authors showed that Ricci curvature $\tilde{S}$ in the direction of $\xi$ is given by

$$
\tilde{S}(\xi, \xi)=-2 n \alpha^{2}+\operatorname{tr} \tilde{h}^{2} .
$$

We recall that the curvature tensor of a 3-dimensional pseudo-Riemannian manifold satisfies

$$
\begin{aligned}
\tilde{R}(X, Y) Z= & \tilde{g}(Y, Z) \tilde{Q} X-\tilde{g}(X, Z) \tilde{Q} Y+\tilde{g}(\tilde{Q} Y, Z) X-\tilde{g}(\tilde{Q} X, Z) Y \\
& -\frac{\tau}{2}(\tilde{g}(Y, Z) X-\tilde{g}(X, Z) Y)
\end{aligned}
$$




\section{Almost $\alpha$-para-Kenmotsu $(\tilde{\kappa}, \tilde{\mu}, \tilde{\nu})$-spaces}

Firstly, let us recall the following theorem which is exactly the same as almost Kenmotsu manifolds [9], where $\tilde{h}=0$, it is certainly $\tilde{h}^{2}=0$.

Theorem 3.1. Let $M^{2 n+1}$ be an almost $\alpha$-para-Kenmotsu manifold with $\tilde{h}=0$. Then $M^{2 n+1}$ is locally a warped product $M_{1} \times_{f^{2}} M_{2}$, where $M_{2}$ is an almost paraKähler manifold, $M_{1}$ is an open interval with coordinate t, and $f^{2}=w e^{2 \alpha t}$ for some positive constant $w$.

Now, we give some properties for later use.

Lemma 3.1. Let $\left(M^{2 n+1}, \tilde{\varphi}, \xi, \eta, \tilde{g}\right)$ be an almost $\alpha$-para-Kenmotsu manifold, then, for any orthonormal frame $X_{i}, i=1, \cdots, 2 n+1$, the following identities hold:

$$
\begin{gathered}
\sum_{i=1}^{2 n+1} \varepsilon_{i}\left(\tilde{\nabla}_{X_{i}} \tilde{\varphi} \tilde{h}\right) X_{i}=\tilde{Q} \xi+2 n \alpha^{2} \xi, \\
\sum_{i=1}^{2 n+1} \varepsilon_{i}\left(\tilde{\nabla}_{X_{i}} \tilde{\varphi}\right) X_{i}=0 .
\end{gathered}
$$

Proof. Let $X_{i}(i=1, \cdots, 2 n+1)$ be an orthonormal frame. For any vector field $X$, putting $X=X_{i}$, replacing $Y$ by $\tilde{\varphi} X$ in (2.6), taking the inner product with $X=X_{i}$, by using $\tilde{h} \xi=\tilde{\varphi} \xi=0, \operatorname{tr}(\tilde{\varphi} \tilde{h})=0$, the symmetry of $\tilde{\nabla}_{X_{i}} \tilde{\varphi} \tilde{h}$, and the skew-symmetry of $\tilde{\varphi}$ we get

$$
\begin{aligned}
& \tilde{g}(\tilde{Q} \xi, \tilde{\varphi} X) \\
= & \sum_{i=1}^{2 n+1} \varepsilon_{i} \tilde{g}\left(\tilde{R}\left(X_{i}, \tilde{\varphi} X\right) \xi, X_{i}\right) \\
= & \sum_{i=1}^{2 n+1} \varepsilon_{i}\left\{\alpha \eta\left(X_{i}\right) \tilde{g}\left(\alpha \tilde{\varphi} X-\tilde{\varphi} \tilde{h} \tilde{\varphi} X, X_{i}\right)+\tilde{g}\left(\left(\tilde{\nabla}_{X_{i}} \tilde{\varphi} \tilde{h}\right) \tilde{\varphi} X, X_{i}\right)-\tilde{g}\left(\left(\tilde{\nabla}_{\tilde{\varphi} X} \tilde{\varphi} \tilde{h}\right) X_{i}, X_{i}\right)\right\} \\
= & \sum_{i=1}^{2 n+1} \varepsilon_{i} \tilde{g}\left(\left(\tilde{\nabla}_{X_{i}} \tilde{\varphi} \tilde{h}\right) \tilde{\varphi} X, X_{i}\right) .
\end{aligned}
$$

Thus the above equality reduces to

$$
\tilde{\varphi} \tilde{Q} \xi=\sum_{i=1}^{2 n+1} \varepsilon_{i} \tilde{\varphi}\left(\tilde{\nabla}_{X_{i}} \tilde{\varphi} \tilde{h}\right) X_{i},
$$

Applying $\tilde{\varphi}$ to the above equality, using $\tilde{\varphi}^{2}=\mathrm{Id}-\eta \otimes \xi$ and (2.8), combining with (2.4), we get $\sum_{i=1}^{2 n+1} \varepsilon_{i} \tilde{g}\left(\left(\tilde{\nabla}_{X_{i}} \tilde{\varphi} \tilde{h}\right) X_{i}, \xi\right)=\operatorname{tr} \tilde{h}^{2}$, it follows that

$$
\sum_{i=1}^{2 n+1} \varepsilon_{i}\left(\tilde{\nabla}_{X_{i}} \tilde{\varphi} \tilde{h}\right) X_{i}=\tilde{Q} \xi+2 n \alpha^{2} \xi .
$$


In order to obtain (3.4), we choose a $\tilde{\varphi}$-basis $\left\{E_{i}, \tilde{\varphi} E_{i}, \xi\right\}$, using $(2.6)$ and $\tilde{\nabla} \xi \tilde{\varphi}=0$, we get

$$
\sum_{i=1}^{2 n+1} \varepsilon_{i}\left(\tilde{\nabla}_{X_{i}} \tilde{\varphi}\right) X_{i}=\sum_{i=1}^{n} \varepsilon_{i}\left(\tilde{\nabla}_{E_{i}} \tilde{\varphi}\right) E_{i}-\sum_{i=1}^{n} \varepsilon_{i}\left(\tilde{\nabla}_{\tilde{\varphi} E_{i}} \tilde{\varphi}\right) \tilde{\varphi} E_{i}+\left(\tilde{\nabla}_{\xi} \tilde{\varphi}\right) \xi=0 .
$$

The next lemma concerns almost $\alpha$-para-Kenmotsu manifolds having the canonical distribution $\mathcal{D}$ with para-Kähler leaves for which the following formula holds [9]:

$$
\left(\tilde{\nabla}_{X} \tilde{\varphi}\right) Y=\tilde{g}(\alpha \tilde{\varphi} X+\tilde{h} X, Y) \xi-\eta(Y)(\alpha \tilde{\varphi} X+\tilde{h} X) .
$$

Lemma 3.2. Let $\left(M^{2 n+1}, \tilde{\varphi}, \xi, \eta, \tilde{g}\right)$ be an almost $\alpha$-para-Kenmotsu manifold and assume that the distribution $\mathcal{D}$ has para-Kähler leaves, then, for any orthonormal frame $X_{i}, i=1, \cdots, 2 n+1$, we have

$$
\sum_{i=1}^{2 n+1} \varepsilon_{i}\left(\tilde{\nabla}_{X_{i}} \tilde{h}\right) X_{i}=\tilde{\varphi} \tilde{Q} \xi
$$

Proof. Since

$$
\begin{gathered}
\tilde{\nabla}_{X} \tilde{h} \tilde{\varphi} Y=\left(\tilde{\nabla}_{X} \tilde{h}\right) \tilde{\varphi} Y+\tilde{h}\left(\tilde{\nabla}_{X} \tilde{\varphi}\right) Y+\tilde{h} \tilde{\varphi} \tilde{\nabla}_{X} Y, \\
\tilde{\nabla}_{X} \tilde{\varphi} \tilde{h} Y=\tilde{\varphi}\left(\tilde{\nabla}_{X} \tilde{h}\right) Y+\tilde{\varphi} \tilde{h}\left(\tilde{\nabla}_{X} Y\right)+\left(\tilde{\nabla}_{X} \tilde{\varphi}\right) \tilde{h} Y,
\end{gathered}
$$

By (3.5)-(3.6) and $\tilde{\varphi} \tilde{h}=-\tilde{h} \tilde{\varphi}$, we get

$$
\begin{aligned}
\left(\tilde{\nabla}_{X} \tilde{h}\right) \tilde{\varphi} Y+\tilde{\varphi}\left(\tilde{\nabla}_{X} \tilde{h}\right) Y & =-\tilde{h}\left(\tilde{\nabla}_{X} \tilde{\varphi}\right) Y-\left(\tilde{\nabla}_{X} \tilde{\varphi}\right) \tilde{h} Y \\
& =\eta(Y)\left(\alpha \tilde{h} \tilde{\varphi} X+\tilde{h}^{2} X\right)-\tilde{g}(\alpha \tilde{\varphi} X+\tilde{h} X, \tilde{h} Y) \xi .
\end{aligned}
$$

Taking $X=Y=X_{i}$ in (3.7), summing on $i$ and using $\operatorname{tr}(\tilde{h} \tilde{\varphi})=0$ and $\tilde{h} \xi=0$, we get

$$
\sum_{i=1}^{2 n+1} \varepsilon_{i}\left\{\left(\tilde{\nabla}_{X_{i}} \tilde{h}\right) \tilde{\varphi} X_{i}+\tilde{\varphi}\left(\tilde{\nabla}_{X_{i}} \tilde{h}\right) X_{i}\right\}=-\left(\operatorname{tr} \tilde{h}^{2}\right) \xi
$$

By (3.1), and using (3.4) we get

$$
\begin{aligned}
\tilde{Q} \xi+2 n \alpha^{2} \xi & =\sum_{i=1}^{2 n+1} \varepsilon_{i}\left(\tilde{\nabla}_{X_{i}} \tilde{\varphi} \tilde{h}\right) X_{i}=-\sum_{i=1}^{2 n+1} \varepsilon_{i}\left(\tilde{\nabla}_{X_{i}} \tilde{h} \tilde{\varphi}\right) X_{i} \\
& =-\sum_{i=1}^{2 n+1} \varepsilon_{i}\left\{\left(\tilde{\nabla}_{X_{i}} \tilde{h}\right) \tilde{\varphi} X_{i}+\tilde{h}\left(\tilde{\nabla}_{X_{i}} \tilde{\varphi}\right) X_{i}\right\} \\
& =-\sum_{i=1}^{2 n+1} \varepsilon_{i}\left(\tilde{\nabla} X_{X_{i}} \tilde{h}\right) \tilde{\varphi} X_{i} .
\end{aligned}
$$


Substituting (3.3) into (3.8) we obtain

$$
\sum_{i=1}^{2 n+1} \varepsilon_{i} \tilde{\varphi}\left(\tilde{\nabla}_{X_{i}} \tilde{h}\right) X_{i}=\tilde{Q} \xi+\left(2 n \alpha^{2}-\operatorname{tr} \tilde{h}^{2}\right) \xi
$$

finally, we get the required result acting by $\tilde{\varphi}$ and using $\sum_{i=1}^{2 n+1} \varepsilon_{i} \tilde{g}\left(\left(\tilde{\nabla}_{X_{i}} \tilde{h}\right) X_{i}, \xi\right)=$ 0 , which, by direct calculation, follows from the fact that $\tilde{g}\left(\tilde{\varphi} \tilde{h}^{2} X_{i}, X_{i}\right)=0$ and $\operatorname{tr}(\tilde{h} \tilde{\varphi})=0$.

Next we study almost $\alpha$-para-Kenmotsu manifolds under assumption that the curvature satisfies $(\tilde{\kappa}, \tilde{\mu}, \tilde{\nu})$-nullity condition

$$
\tilde{R}(X, Y) \xi=\eta(Y) B X-\eta(X) B Y,
$$

where $B$ is Jacobi operator of $\xi$, that is to say $B X=\tilde{R}(X, \xi) \xi=\tilde{\kappa} \tilde{\varphi}^{2} X+\tilde{\mu} \tilde{h} X+$ $\tilde{\nu} \tilde{\varphi} \tilde{h} X$, for $\tilde{\kappa}, \tilde{\mu}, \tilde{\nu} \in R_{\eta}\left(M^{2 n+1}\right)$. Particularly $B \xi=0$. If $\tilde{\mu}=0$ or $\tilde{h}=0$ and $\tilde{\nu}=0$ or $\tilde{\varphi} \tilde{h}=0$, the $(\tilde{\kappa}, \tilde{\mu}, \tilde{\nu})$-nullity distribution is reduced to the well-known $\tilde{\kappa}$-nullity distribution $N(\tilde{\kappa})$. The $(\tilde{\kappa}, \tilde{\mu}, \tilde{\nu})$-nullity condition $(3.10)$ is obtained by requiring that $\xi$ belong to some $N(\tilde{\kappa}, \tilde{\mu}, \tilde{\nu})$. If almost $\alpha$-para-Kenmotsu manifold satisfies (3.10), then the manifold is said to be an almost $\alpha$-para-Kenmotsu $(\tilde{\kappa}, \tilde{\mu}, \tilde{\nu})$-space. We observe that, in an almost $\alpha$-para-Kenmotsu manifold, if $\xi \in N(\tilde{\kappa}, \tilde{\mu}, \tilde{\nu}),(3.10)$ and (2.5) implies $\tilde{\varphi} \tilde{h}$ is a Codazzi tensor, that is to say, $\left(\tilde{\nabla}_{X} \tilde{\varphi} \tilde{h}\right) Y-\left(\tilde{\nabla}_{Y} \tilde{\varphi} \tilde{h}\right) X=0$, for any $X, Y \in \mathcal{D}$.

Proposition 3.1. [9] Let $\left(M^{2 n+1}, \tilde{\varphi}, \xi, \eta, \tilde{g}\right)$ be an almost $\alpha$-para-Kenmotsu $(\kappa, \mu, \nu)$ space, then the following identities hold:

$$
\begin{gathered}
\tilde{h}^{2}=\left(\tilde{\kappa}+\alpha^{2}\right) \tilde{\varphi}^{2}, \\
\tilde{\nabla}_{\xi} \tilde{h}=-(2 \alpha+\tilde{\nu}) \tilde{h}+\tilde{\mu} \tilde{h} \tilde{\varphi} \\
\xi(\tilde{\kappa})=-2(2 \alpha+\tilde{\nu})\left(\tilde{\kappa}+\alpha^{2}\right), \\
\tilde{Q} \xi=2 n \tilde{\kappa} \xi .
\end{gathered}
$$

Lemma 3.3. Let $\left(M^{3}, \tilde{\varphi}, \xi, \eta, \tilde{g}\right)$ be an almost $\alpha$-para-Kenmotsu $(\tilde{\kappa}, \tilde{\mu}, \tilde{\nu}=$ const. $)$ spaces, then one has:

$$
\begin{gathered}
\tilde{Q} X=\left(-\tilde{\kappa}+\frac{\tau}{2}\right) X+\left(3 \tilde{\kappa}-\frac{\tau}{2}\right) \eta(X) \xi+\tilde{\mu} \tilde{h} X+\tilde{\nu} \tilde{\varphi} \tilde{h} X, \\
\tilde{h} \operatorname{grad} \tilde{\mu}+\tilde{\varphi} \tilde{h} \operatorname{grad} \tilde{\nu}=\operatorname{grad} \tilde{\kappa}-\xi(\tilde{\kappa}) \xi
\end{gathered}
$$

where $\tilde{Q}$ is the Ricci operator of $M . \tau$ denotes scalar curvature of $M$ and $\tilde{l}=$ $\tilde{R}(\cdot, \xi) \xi$. 
Proof. Let $Y=Z=\xi$ in (2.9) and using (3.10), we can easily obtain (3.15).

By using the well known formula

$$
\frac{1}{2} \operatorname{grad} \tau=\sum_{i=1}^{3} \varepsilon_{i}\left(\nabla_{X_{i}} \tilde{Q}\right) X_{i}
$$

for any orthonormal frames $X_{i}, i=1,2,3$, using $(2.2)$ and (3.15), since $\operatorname{tr} \tilde{h}=\operatorname{tr} \tilde{h} \tilde{\varphi}=$ 0 , we have

$$
\begin{aligned}
\frac{1}{2} \operatorname{grad} \tau= & \sum_{i=1}^{3} \varepsilon_{i}\left(\nabla_{X_{i}} Q\right) X_{i}=\sum_{i=1}^{3} \varepsilon_{i}\left(\nabla_{X_{i}} Q X_{i}-Q \nabla_{X_{i}} X_{i}\right) \\
= & \sum_{i=1}^{3} \varepsilon_{i}\left\{\nabla_{X_{i}}\left[\left(-\tilde{\kappa}+\frac{\tau}{2}\right) X_{i}+\left(3 \tilde{\kappa}-\frac{\tau}{2}\right) \eta\left(X_{i}\right) \xi+\tilde{\mu} \tilde{h} X_{i}+\tilde{\nu} \tilde{\varphi} \tilde{h} X_{i}\right]\right. \\
& \left.-\left[\left(-\tilde{\kappa}+\frac{\tau}{2}\right) \nabla_{X_{i}} X_{i}+\left(3 \tilde{\kappa}-\frac{\tau}{2}\right) \eta\left(\nabla_{X_{i}} X_{i}\right) \xi+\tilde{\mu} \tilde{h} \nabla_{X_{i}} X_{i}+\tilde{\nu} \tilde{\varphi} \tilde{h} \nabla_{X_{i}} X_{i}\right]\right\} \\
= & \sum_{i=1}^{3} \varepsilon_{i}\left\{X_{i}\left(-\tilde{\kappa}+\frac{\tau}{2}\right) X_{i}+X_{i}\left(3 \tilde{\kappa}-\frac{\tau}{2}\right) \eta\left(X_{i}\right) \xi+X_{i}(\tilde{\mu}) \tilde{h} X_{i}+X_{i}(\tilde{\nu}) \tilde{\varphi} \tilde{h} X_{i}\right\} \\
& +\sum_{i=1}^{3} \varepsilon_{i}\left\{\tilde{\mu}\left(\tilde{\nabla}_{X_{i}} \tilde{h}\right) X_{i}+\tilde{\nu}\left(\tilde{\nabla}_{X_{i}} \tilde{\varphi} \tilde{h}\right) X_{i}\right\} \\
= & -\operatorname{grad} \tilde{\kappa}+\frac{1}{2} \operatorname{grad} \tau+\tilde{h} \operatorname{grad} \tilde{\mu}+\tilde{\varphi} \tilde{h} \operatorname{grad} \tilde{\nu}+\left[3 \xi(\tilde{\kappa})-\frac{1}{2} \xi(\tau)\right] \xi \\
& +\sum_{i=1}^{3} \varepsilon_{i}\left\{\tilde{\mu}\left(\tilde{\nabla} X_{i} \tilde{h}\right) X_{i}+\tilde{\nu}\left(\tilde{\nabla} X_{i} \tilde{\varphi} \tilde{h}\right) X_{i}\right\} .
\end{aligned}
$$

Thus, using (3.1), (3.2) and (3.14) we get

$$
\sum_{i=1}^{3} \varepsilon_{i}\left(\tilde{\nabla}_{X_{i}} \tilde{h}\right) X_{i}=0
$$

and

$$
\sum_{i=1}^{3} \varepsilon_{i}\left(\tilde{\nabla}_{X_{i}} \tilde{\varphi} \tilde{h}\right) X_{i}=2\left(\tilde{\kappa}+\alpha^{2}\right) \xi
$$

Using these two equalities in (3.15), one has

$$
\xi(\tilde{\kappa}) \xi-\operatorname{grad} \tilde{\kappa}+\tilde{h} \operatorname{grad} \tilde{\mu}+\tilde{\varphi} \tilde{h} \operatorname{grad} \tilde{\nu}+\xi\left(2 \tilde{\kappa}-\frac{1}{2} \tau\right) \xi+2\left(\alpha^{2}+\tilde{\kappa}\right) \tilde{\nu} \xi=0 .
$$

Since the vector field $\xi(\tilde{\kappa}) \xi-\operatorname{grad} \tilde{\kappa}+\tilde{h} \operatorname{grad} \tilde{\mu}+\tilde{\varphi} \tilde{h} \operatorname{grad} \tilde{\nu}$ is orthogonal to $\xi$, (3.16) follows. 
Proposition 3.2. Let $\left(M^{3}, \tilde{\varphi}, \xi, \eta, \tilde{g}\right)$ be an almost $\alpha$-para-Kenmotsu manifold. If $M$ is $\eta$-Einstein, then $\xi \in N(\tilde{\kappa})$ for some function $\tilde{\kappa}$.

Proof. By (2.7), choosing the $\tilde{\varphi}$-basis $\{\xi, e, \tilde{\varphi} e\}$, we get $\tilde{Q} \xi=(a+b) \xi$ and $\tau=$ $\tilde{g}(\xi, \xi)+\tilde{g}(\tilde{Q} e, e)+\tilde{g}(\tilde{Q} \tilde{\varphi} e, \tilde{\varphi} e)=3 a+b$. Let $Z=\xi$ in (??) and using (2.7), we can easily obtain $\tilde{R}(X, Y) \xi=\frac{a+b}{2}(\eta(Y) X-\eta(X) Y)$, thus $\xi \in N\left(\frac{a+b}{2}\right)$.

Corollary 3.1. Let $\left(M^{3}, \tilde{\varphi}, \xi, \eta, \tilde{g}\right)$ be an almost $\alpha$-para-Kenmotsu manifold. If $M$ is $\xi \in N(\tilde{\kappa})$, then $M$ is $\eta$-Einstein.

Proof. By Lemma 3.3, we get $\tilde{Q} X=\left(-\tilde{\kappa}+\frac{\tau}{2}\right) X+\left(3 \tilde{\kappa}-\frac{\tau}{2}\right) \eta(X) \xi$, it is simply to get that $\mathrm{M}$ is $\eta$-Einstein.

If $\tilde{h}=0$, by (2.5), we get $\tilde{R}(X, Y) \xi=-\alpha^{2}(\eta(Y) X-\eta(X) Y)$, thus $\xi \in N\left(-\alpha^{2}\right)$, by Corollary 3.1, it follows that $\mathrm{M}$ is $\eta$-Einstein. Therefore, from now on, we will restrict our investigations mainly on the more meaningful case $\tilde{h} \neq 0$. I. K. Erken, P. Dacko and C. Murathan analyzed the different possibilities for the tensor field $\tilde{h}$ in [9]. If $\tilde{h}$ has

$$
\left(\begin{array}{ccc}
\tilde{\lambda} & 0 & 0 \\
0 & -\tilde{\lambda} & 0 \\
0 & 0 & 0
\end{array}\right)
$$

with respect to a local orthonormal $\tilde{\varphi}$-basis $\{X, \tilde{\varphi} X, \xi\}$, the authors called the operator $\tilde{h}$ is of $\mathfrak{h}_{1}$ type.

If $\tilde{h}$ has

$$
\left(\begin{array}{lll}
0 & 0 & 0 \\
1 & 0 & 0 \\
0 & 0 & 0
\end{array}\right)
$$

with respect to a pseudo orthonormal basis $\left\{e_{1}, e_{2}, e_{3}\right\}$, the authors called the operator $\tilde{h}$ is of $\mathfrak{h}_{2}$ type.

If $\tilde{h}$ has

$$
\left(\begin{array}{ccc}
0 & \tilde{\lambda} & 0 \\
-\tilde{\lambda} & 0 & 0 \\
0 & 0 & 0
\end{array}\right)
$$

with respect to a local orthonormal $\tilde{\varphi}$-basis $\{X, \tilde{\varphi} X, \xi\}$, in this case. the authors called the operator $\tilde{h}$ is of $\mathfrak{h}_{3}$ type.

It follows that $\tilde{h}^{2} X=\tilde{\lambda}^{2} X$ if $\tilde{h}$ is of $\mathfrak{h}_{1}$ type and $\tilde{h}^{2} X=-\tilde{\lambda}^{2} X$ if $\tilde{h}$ is of $\mathfrak{h}_{3}$ type, but $\tilde{h}^{2} X=0$ if $\tilde{h}$ is of $\mathfrak{h}_{2}$ type though $\tilde{h} \neq 0$, and there are examples of 3 -dimensional almost $\alpha$-para-Kenmotsu manifold of this case [9]. In this paper, we manly discuss the case $\tilde{h}^{2} \neq 0$, that is, $\tilde{\kappa}+\alpha^{2} \neq 0$. 
Lemma 3.4. Let $\left(M^{3}, \tilde{\varphi}, \xi, \eta, \tilde{g}\right)$ be an almost $\alpha$-para-Kenmotsu $(\tilde{\kappa}, \tilde{\mu}, \tilde{\nu}=$ const. $)$ space with $\tilde{h}$ is of $\mathfrak{h}_{1}$ type. Then, for any point $p \in M$, there exist a neighborhood $U$ of $p$ and a $\tilde{\varphi}$-basis $\{X, \tilde{\varphi} X, \xi\}$ defined on $U$, such that

$$
\tilde{h} X=\tilde{\lambda} X, \quad \tilde{h} \tilde{\varphi} X=-\tilde{\lambda} \tilde{\varphi} X, \quad \tilde{h} \xi=0, \quad \tilde{\lambda}=\sqrt{\tilde{\kappa}+\alpha^{2}}
$$

at any point $q \in U$. Moreover, setting $A=X(\tilde{\lambda})$ and $B=\tilde{\varphi} X(\tilde{\lambda})$ on $U$ the following formulas are true:

$$
\begin{gathered}
\tilde{\nabla}_{X} \xi=\alpha X+\tilde{\lambda} \tilde{\varphi} X, \quad \tilde{\nabla}_{\tilde{\varphi} X} \xi=\alpha \tilde{\varphi} X-\tilde{\lambda} X \\
\tilde{\nabla}_{\xi} X=-\frac{\tilde{\mu}}{2} \tilde{\varphi} X, \quad \tilde{\nabla}_{\xi} \tilde{\varphi} X=-\frac{\tilde{\mu}}{2} X \\
\tilde{\nabla}_{X} X=\alpha \xi-\frac{B}{2 \tilde{\lambda}} \tilde{\varphi} X, \quad \tilde{\nabla}_{\tilde{\varphi} X} \tilde{\varphi} X=-\alpha \xi-\frac{A}{2 \tilde{\lambda}} X \\
\tilde{\nabla}_{\tilde{\varphi} X} X=-\tilde{\lambda} \xi-\frac{A}{2 \tilde{\lambda}} \tilde{\varphi} X, \tilde{\nabla}_{X} \tilde{\varphi} X=-\tilde{\lambda} \xi-\frac{B}{2 \tilde{\lambda}} X \\
{[\xi, X]=-\alpha X-\left(\tilde{\lambda}+\frac{\tilde{\mu}}{2}\right) \tilde{\varphi} X,[\xi, \tilde{\varphi} X]=\left(\tilde{\lambda}-\frac{\tilde{\mu}}{2}\right) X-\alpha \tilde{\varphi} X} \\
{[X, \tilde{\varphi} X]=-\frac{B}{2 \tilde{\lambda}} X+\frac{A}{2 \tilde{\lambda}} \tilde{\varphi} X .} \\
\tilde{h} \operatorname{grad} \tilde{\mu}=\operatorname{grad} \tilde{\kappa}-\xi(\tilde{\kappa}) \xi
\end{gathered}
$$

Proof. By [9] we know that if $\tilde{h}$ is of $\mathfrak{h}_{1}$ type with respect to a $\tilde{\varphi}$-basis $\{X, \tilde{\varphi} X, \xi\}$ such that $\tilde{h} X=\tilde{\lambda} X, \tilde{h} \tilde{\varphi} X=-\tilde{\lambda} \tilde{\varphi} X$, and by (3.11), we get $\tilde{\lambda}=\sqrt{\tilde{\kappa}+\alpha^{2}}$. Similar as the proof of [16], we get Lemma 3.4.

Similarly as Lemma 3.4, we get the following Lemma.

Lemma 3.5. Let $\left(M^{3}, \tilde{\varphi}, \xi, \eta, \tilde{g}\right)$ be an almost $\alpha$-para-Kenmotsu $(\tilde{\kappa}, \tilde{\mu}, \tilde{\nu}=$ const. $)$ space with $\tilde{h}$ is of $\mathfrak{h}_{3}$ type. Then, for any point $p \in M$, there exist a neighborhood $U$ of $p$ and a $\tilde{\varphi}$-basis $\{X, \tilde{\varphi} X, \xi\}$ defined on $U$, such that

$$
\tilde{h} X=\tilde{\lambda} \tilde{\varphi} X, \quad \tilde{h} \tilde{\varphi} X=-\tilde{\lambda} X, \quad \tilde{h} \xi=0, \quad \tilde{\lambda}=\sqrt{-\left(\tilde{\kappa}+\alpha^{2}\right)}
$$

at any point $q \in U$. Moreover, setting $A=X(\tilde{\lambda})$ and $B=\tilde{\varphi} X(\tilde{\lambda})$ on $U$ the following formulas are true:

$$
\tilde{\nabla}_{X} \xi=(\alpha+\tilde{\lambda}) X, \quad \tilde{\nabla}_{\tilde{\varphi} X} \xi=(\alpha-\tilde{\lambda}) \tilde{\varphi} X
$$




$$
\begin{gathered}
\tilde{\nabla}_{\xi} X=-\frac{\tilde{\mu}}{2} \tilde{\varphi} X, \quad \tilde{\nabla}_{\xi} \tilde{\varphi} X=-\frac{\tilde{\mu}}{2} X, \\
\tilde{\nabla}_{X} X=(\alpha+\tilde{\lambda}) \xi-\frac{B}{2 \tilde{\lambda}} \tilde{\varphi} X, \quad \tilde{\nabla}_{\tilde{\varphi} X} \tilde{\varphi} X=(\tilde{\lambda}-\alpha) \xi-\frac{A}{2 \tilde{\lambda}} X, \\
\tilde{\nabla}_{\tilde{\varphi} X} X=-\frac{A}{2 \tilde{\lambda}} \tilde{\varphi} X, \tilde{\nabla}_{X} \tilde{\varphi} X=-\frac{B}{2 \tilde{\lambda}} X \\
{[\xi, X]=-(\alpha+\tilde{\lambda}) X-\frac{\tilde{\mu}}{2} \tilde{\varphi} X,[\xi, \tilde{\varphi} X]=-\frac{\tilde{\mu}}{2} X+(\tilde{\lambda}-\alpha) \tilde{\varphi} X,} \\
{[X, \tilde{\varphi} X]=-\frac{B}{2 \tilde{\lambda}} X+\frac{A}{2 \tilde{\lambda}} \tilde{\varphi} X .} \\
\tilde{h} \operatorname{grad} \tilde{\mu}=\operatorname{grad} \tilde{\kappa}-\xi(\tilde{\kappa}) \xi .
\end{gathered}
$$

4. Almost $\alpha$-para-Kenmotsu $(\tilde{\kappa}, \tilde{\mu}, \tilde{\nu}=$ const. $)$-space with $d \tilde{\kappa} \wedge \eta=0$

Locally, an almost $\alpha$-para-Kenmotsu $(\tilde{\kappa}, \tilde{\mu}, \tilde{\nu}=$ const. $)$-space with $\tilde{h}$ is of $\mathfrak{h}_{1}$ type and $d \tilde{\kappa} \wedge \eta=0$ can be described as follows.

Theorem 4.1. Let $\left(M^{3}, \tilde{\varphi}, \xi, \eta, \tilde{g}\right)$ be an almost $\alpha$-para-Kenmotsu $(\tilde{\kappa}, \tilde{\mu}, \tilde{\nu}=$ const. $)$ space with $\tilde{h}$ is of $\mathfrak{h}_{1}$ type and $d \tilde{\kappa} \wedge \eta=0$. Then, in a neighbourhood $U$ of every point $p \in M$, there exist coordinates $x, y, z$ and an orthonormal frame $\{X, \tilde{\varphi} X, \xi\}$ of eigenvectors of $\tilde{h}$ with $\tilde{h} X=\tilde{\lambda} X$, such that on $U \tilde{\kappa}, \tilde{\mu}$ only depends on $z$ and

$$
X=\frac{\partial}{\partial x}, \tilde{\varphi} X=\frac{\partial}{\partial y}, \xi=a \frac{\partial}{\partial x}+b \frac{\partial}{\partial y}+\frac{\partial}{\partial z},
$$

and the tensor fields $\tilde{\varphi}, \tilde{g}, \tilde{h}$ are given by the relations:

$\tilde{g}=\left(\begin{array}{ccc}-1 & 0 & a \\ 0 & 1 & -b \\ a & -b & 1-a^{2}+b^{2}\end{array}\right), \tilde{\varphi}=\left(\begin{array}{ccc}0 & 1 & -b \\ 1 & 0 & -a \\ 0 & 0 & 0\end{array}\right), \tilde{h}=\left(\begin{array}{ccc}\tilde{\lambda} & 0 & -a \tilde{\lambda} \\ 0 & -\tilde{\lambda} & b \tilde{\lambda} \\ 0 & 0 & 0\end{array}\right)$.

where $a=\alpha x+\left(\frac{\tilde{\mu}}{2}-\tilde{\lambda}\right) y+f(z), b=\left(\frac{\tilde{\mu}}{2}+\tilde{\lambda}\right) x-\alpha y-g(z), f(z), g(z)$ are arbitrary smooth functions of $z, \alpha$ is a constant value.

Proof. The condition $d \tilde{\kappa} \wedge \eta=0$ and (3.28) means that $d \tilde{\mu} \wedge \eta=0$, since $\tilde{h} \neq 0$ and $\operatorname{ker} \tilde{h}=\operatorname{Span}\{\xi\}$. Moreover, we have $E(\tilde{\lambda})=0$ for all $E \in \mathcal{D}$. By lemma 3.4, we get that for any point $p \in M$, there exist a neighborhood $U$ of $p$ and a $\tilde{\varphi}$-basis $\{X, \tilde{\varphi} X, \xi\}$ defined on $U$, such that $\tilde{h} X=\tilde{\lambda} X, \tilde{h} \tilde{\varphi} X=-\tilde{\lambda} \tilde{\varphi} X, \tilde{\lambda}=\sqrt{\tilde{\kappa}+\alpha^{2}}$. 
Hence $A=X(\tilde{\lambda})=B=\tilde{\varphi} X(\tilde{\lambda})=0$, that is to say, by Lemma 3.4, we get that $[X, \tilde{\varphi} X]=0$. So, fixed the point $p \in M$, there exist coordinates $(x, y, t)$ on an open neighbourhood $V$ of $p$ such that

$$
X=\frac{\partial}{\partial x}, \tilde{\varphi} X=\frac{\partial}{\partial y}, \xi=a \frac{\partial}{\partial x}+b \frac{\partial}{\partial y}+c \frac{\partial}{\partial t},
$$

where $a, b$ and $c$ are smooth functions on $V$ with $c \neq 0$ everywhere. Since we get $[X, \xi] \in \mathcal{D}$ and $[X, \xi] \in \mathcal{D}$ for any $X \in \mathcal{D}$ by $d \eta=0$, we obtain that $\frac{\partial c}{\partial x}=0$ and $\frac{\partial c}{\partial y}=0$. Therefore, if we consider on $V$ the linearly independent vector fields $X, \tilde{\varphi} X$ and $Z=c \frac{\partial}{\partial t}$, we have

$$
[X, \tilde{\varphi} X]=0,[X, Z]=0,[\tilde{\varphi} X, Z]=0 .
$$

This implies that there exists a coordinate system $\{U,(x, y, z)\}$ around $p$ in $V$ such that $X=\frac{\partial}{\partial x}, \tilde{\varphi} X=\frac{\partial}{\partial y}$ and $Z=\frac{\partial}{\partial z}$. Thus, on the open set $U$ we have $\xi=a \frac{\partial}{\partial x}+b \frac{\partial}{\partial y}+\frac{\partial}{\partial z}$. From (3.13) and (3.21), we get that $\xi(\tilde{\lambda})=-(2 \alpha+\tilde{\nu}) \tilde{\lambda}$, and since $A=X(\tilde{\lambda})=B=\tilde{\varphi} X(\tilde{\lambda})=0$, it follows that $\tilde{\lambda}=c e^{-(2 \alpha+\tilde{\nu}) z}$, and $\tilde{\kappa}=\tilde{\lambda}^{2}-\alpha^{2}=c^{2} e^{-2(2 \alpha+\tilde{\nu}) z}-\alpha^{2}$ for some real constant $c>0$. Since $d \tilde{\mu} \wedge \eta=0$, we get that $\tilde{\mu}=\tilde{\mu}(z)$. Next, we need to compute the functions $a, b$. To this end,

$$
[\xi, X]=-\frac{\partial a}{\partial x} \frac{\partial}{\partial x}-\frac{\partial b}{\partial x} \frac{\partial}{\partial y}, \quad[\xi, \tilde{\varphi} X]=-\frac{\partial a}{\partial y} \frac{\partial}{\partial x}-\frac{\partial b}{\partial y} \frac{\partial}{\partial y}
$$

And by Lemma 3.4, we obtain

$$
[\xi, X]=-\alpha \frac{\partial}{\partial x}-\left(\tilde{\lambda}+\frac{\tilde{\mu}}{2}\right) \frac{\partial}{\partial y}, \quad[\xi, \tilde{\varphi} X]=\left(\tilde{\lambda}-\frac{\tilde{\mu}}{2}\right) \frac{\partial}{\partial x}-\alpha \frac{\partial}{\partial y},
$$

The comparison of these relations with the previous leads to

$$
\frac{\partial a}{\partial x}=\alpha, \quad \frac{\partial a}{\partial y}=\frac{\tilde{\mu}}{2}-\tilde{\lambda}, \quad \frac{\partial b}{\partial x}=\tilde{\lambda}+\frac{\tilde{\mu}}{2}, \quad \frac{\partial b}{\partial y}=\alpha .
$$

By integration of these system, considering $\tilde{\lambda}, \tilde{\mu}$ functions depending only on $z$, we get $a=\alpha x+\left(\frac{\tilde{\mu}}{2}-\tilde{\lambda}\right) y+f(z), b=\left(\frac{\tilde{\mu}}{2}+\tilde{\lambda}\right) x-\alpha y-g(z), f(z), g(z)$ are arbitrary smooth functions of $z$.

We will continue calculate the tensor fields $\eta, \tilde{\varphi}, \tilde{g}$ and $\tilde{h}$ with respect to the basis $\frac{\partial}{\partial x}, \frac{\partial}{\partial y}, \frac{\partial}{\partial z}$. The expression of the 1-form $\eta=d z$ immediately follows from $\eta(\xi)=1, \eta(X)=\eta(\tilde{\varphi} X)=0$. For the components of $\tilde{g}_{i j}$ of the pseudo-Riemannian metric, we have

$$
\begin{gathered}
\tilde{g}_{11}=\tilde{g}\left(\frac{\partial}{\partial x}, \frac{\partial}{\partial x}\right)=\tilde{g}(X, X)=-1, \quad \tilde{g}_{22}=\tilde{g}\left(\frac{\partial}{\partial y}, \frac{\partial}{\partial y}\right)=\tilde{g}(\tilde{\varphi} X, \tilde{\varphi} X)=1, \\
\tilde{g}_{33}=\tilde{g}\left(\frac{\partial}{\partial z}, \frac{\partial}{\partial z}\right)=\tilde{g}\left(\xi-a \frac{\partial}{\partial x}-b \frac{\partial}{\partial y}, \xi-a \frac{\partial}{\partial x}-b \frac{\partial}{\partial y}\right)=1-a^{2}+b^{2} .
\end{gathered}
$$




$$
\begin{gathered}
\tilde{g}_{12}=\tilde{g}_{21}=\tilde{g}\left(\frac{\partial}{\partial x}, \frac{\partial}{\partial y}\right)=\tilde{g}(X, \tilde{\varphi} X)=0, \quad \tilde{g}_{13}=\tilde{g}_{31}=\tilde{g}\left(\frac{\partial}{\partial x}, \xi-a \frac{\partial}{\partial x}-b \frac{\partial}{\partial y}\right)=a, \\
\tilde{g}_{23}=\tilde{g}_{32}=\tilde{g}\left(\frac{\partial}{\partial y}, \xi-a \frac{\partial}{\partial x}-b \frac{\partial}{\partial y}\right)=-b,
\end{gathered}
$$

thus the matrix form of $\tilde{g}$ with respect to the basis $\frac{\partial}{\partial x}, \frac{\partial}{\partial y}, \frac{\partial}{\partial z}$ is given by

$$
\tilde{g}=\left(\begin{array}{ccc}
-1 & 0 & a \\
0 & 1 & -b \\
a & -b & 1-a^{2}+b^{2}
\end{array}\right) .
$$

The components of the tensor field $\tilde{\varphi}$ are followed by:

$\tilde{\varphi}\left(\frac{\partial}{\partial x}\right)=\frac{\partial}{\partial y}, \quad \tilde{\varphi}\left(\frac{\partial}{\partial y}\right)=\tilde{\varphi}^{2}\left(\frac{\partial}{\partial x}\right)=\frac{\partial}{\partial x}, \quad \tilde{\varphi}\left(\frac{\partial}{\partial z}\right)=\tilde{\varphi}\left(\xi-a \frac{\partial}{\partial x}-b \frac{\partial}{\partial y}\right)=-a \frac{\partial}{\partial y}-b \frac{\partial}{\partial y}$,

thus the matrix form of $\tilde{\varphi}$ with respect to the basis $\frac{\partial}{\partial x}, \frac{\partial}{\partial y}, \frac{\partial}{\partial z}$ is given by

$$
\left(\begin{array}{ccc}
0 & 1 & -b \\
1 & 0 & -a \\
0 & 0 & 0
\end{array}\right)
$$

The components of the tensor field $\tilde{h}$ with respect to the basis $\frac{\partial}{\partial x}, \frac{\partial}{\partial y}, \frac{\partial}{\partial z}$ are given as follows:

$$
\begin{gathered}
\tilde{h}\left(\frac{\partial}{\partial x}\right)=\tilde{h}(X)=\tilde{\lambda} X=\tilde{\lambda} \frac{\partial}{\partial x}, \tilde{h}\left(\frac{\partial}{\partial y}\right)=\tilde{h} \tilde{\varphi} X=-\tilde{\lambda} \tilde{\varphi} X=-\tilde{\lambda} \frac{\partial}{\partial y}, \\
\tilde{h}\left(\frac{\partial}{\partial z}\right)=\tilde{h}\left(\xi-a \frac{\partial}{\partial x}-b \frac{\partial}{\partial y}\right)=-a \tilde{\lambda} \frac{\partial}{\partial x}+b \tilde{\lambda} \frac{\partial}{\partial y} .
\end{gathered}
$$

Thus the matrix form of $\tilde{h}$ is given by

$$
\left(\begin{array}{ccc}
\tilde{\lambda} & 0 & a \tilde{\lambda} \\
0 & -\tilde{\lambda} & b \tilde{\lambda} \\
0 & 0 & 0
\end{array}\right)
$$

Now we consider the case of $\tilde{h}$ is of $\mathfrak{h}_{3}$ type.

Theorem 4.2. Let $\left(M^{3}, \tilde{\varphi}, \xi, \eta, \tilde{g}\right)$ be an almost $\alpha$-para-Kenmotsu $(\tilde{\kappa}, \tilde{\mu}, \tilde{\nu}=$ const. $)$ space with $\tilde{h}$ is of $\mathfrak{h}_{3}$ type and $d \tilde{\kappa} \wedge \eta=0$. Then, in a neighbourhood $U$ of every point $p \in M$, there exist coordinates $x, y, z$ and an orthonormal frame $\{X, \tilde{\varphi} X, \xi\}$ with $\tilde{h} X=\tilde{\lambda} \tilde{\varphi} X, \tilde{h} \tilde{\varphi} X=-\tilde{\lambda} X$, such that on $U \tilde{\kappa}, \tilde{\mu}$ only depends on $z$ and

$$
X=\frac{\partial}{\partial x}, \tilde{\varphi} X=\frac{\partial}{\partial y}, \xi=a \frac{\partial}{\partial x}+b \frac{\partial}{\partial y}+\frac{\partial}{\partial z},
$$


and the tensor fields $\tilde{\varphi}, \tilde{g}, \tilde{h}$ are given by the relations:

$\tilde{g}=\left(\begin{array}{ccc}-1 & 0 & a \\ 0 & 1 & -b \\ a & -b & 1-a^{2}+b^{2}\end{array}\right), \tilde{\varphi}=\left(\begin{array}{ccc}0 & 1 & -b \\ 1 & 0 & -a \\ 0 & 0 & 0\end{array}\right), \tilde{h}=\left(\begin{array}{ccc}0 & -\tilde{\lambda} & b \tilde{\lambda} \\ \tilde{\lambda} & 0 & -a \tilde{\lambda} \\ 0 & 0 & 0\end{array}\right)$.

where $a=(\alpha+\tilde{\lambda}) x+\frac{\tilde{\mu}}{2} y+f(z), b=\frac{\tilde{\mu}}{2} x+(\alpha-\tilde{\lambda}) y+g(z), f(z), g(z)$ are arbitrary smooth functions of $z$.

Proof. The condition $d \tilde{\kappa} \wedge \eta=0$ and (3.28) means that $d \tilde{\mu} \wedge \eta=0$, since $\tilde{h} \neq 0$ and $\operatorname{ker} \tilde{h}=\operatorname{Span}\{\xi\}$. Moreover, we have $E(\tilde{\lambda})=0$ for all $E \in \mathcal{D}$. By lemma 3.5, we get that for any point $p \in M$, there exist a neighborhood $U$ of $p$ and a $\tilde{\varphi}$-basis $\{X, \tilde{\varphi} X, \xi\}$ defined on $U$, such that $\tilde{h} X=\tilde{\lambda} \tilde{\varphi} X, \tilde{h} \tilde{\varphi} X=-\tilde{\lambda} X, \tilde{\lambda}=\sqrt{-\left(\tilde{\kappa}+\alpha^{2}\right)}$. Hence $A=X(\tilde{\lambda})=B=\tilde{\varphi} X(\tilde{\lambda})=0$, that is to say, by Lemma 3.5, we get that $[X, \tilde{\varphi} X]=0$. So, fixed the point $p \in M$, there exist coordinates $(x, y, t)$ on an open neighbourhood $V$ of $p$ such that

$$
X=\frac{\partial}{\partial x}, \tilde{\varphi} X=\frac{\partial}{\partial y}, \xi=a \frac{\partial}{\partial x}+b \frac{\partial}{\partial y}+c \frac{\partial}{\partial t},
$$

where $a, b$ and $c$ are smooth functions on $V$ with $c \neq 0$ everywhere. Since we get $[X, \xi] \in \mathcal{D}$ and $[X, \xi] \in \mathcal{D}$ for any $X \in \mathcal{D}$ by $d \eta=0$, we obtain that $\frac{\partial c}{\partial x}=0$ and $\frac{\partial c}{\partial y}=0$. Therefore, if we consider on $V$ the linearly independent vector field $X, \tilde{\varphi} X$ and $Z=c \frac{\partial}{\partial t}$, we have

$$
[X, \tilde{\varphi} X]=0,[X, Z]=0,[\tilde{\varphi} X, Z]=0 .
$$

This implies that there exists a coordinate system $\{U,(x, y, z)\}$ around $p$ in $V$ such that $X=\frac{\partial}{\partial x}, \tilde{\varphi} X=\frac{\partial}{\partial y}$ and $Z=\frac{\partial}{\partial z}$. Thus, on the open set $U$ we have $\xi=a \frac{\partial}{\partial x}+b \frac{\partial}{\partial y}+\frac{\partial}{\partial z}$. From (3.13) and (3.21), we get that $\xi(\tilde{\lambda})=-(2 \alpha+\tilde{\nu}) \tilde{\lambda}$, and since $A=X(\tilde{\lambda})=B=\tilde{\varphi} X(\tilde{\lambda})=0$, it follows that $\tilde{\lambda}=c e^{-(2 \alpha+\tilde{\nu}) z}$, and $\tilde{\kappa}=-\tilde{\lambda}^{2}-\alpha^{2}=-c^{2} e^{-2(2 \alpha+\tilde{\nu}) z}-\alpha^{2}$ for some real constant $c>0$. Since $d \tilde{\mu} \wedge \eta=0$, we get that $\tilde{\mu}=\tilde{\mu}(z)$. Next, we need to compute the functions $a, b$. To this end,

$$
[X, \xi]=\frac{\partial a}{\partial x} \frac{\partial}{\partial x}+\frac{\partial b}{\partial x} \frac{\partial}{\partial y}, \quad[\tilde{\varphi} X, \xi]=\frac{\partial a}{\partial y} \frac{\partial}{\partial x}+\frac{\partial b}{\partial y} \frac{\partial}{\partial y}
$$

And by Lemma 3.5, we obtain

$$
[X, \xi]=(\alpha+\tilde{\lambda}) \frac{\partial}{\partial x}+\frac{\tilde{\mu}}{2} \frac{\partial}{\partial y}, \quad[\tilde{\varphi} X, \xi]=\frac{\tilde{\mu}}{2} \frac{\partial}{\partial x}+(\alpha-\tilde{\lambda}) \frac{\partial}{\partial y} .
$$

The comparison of these relations with the previous leads to

$$
\frac{\partial a}{\partial x}=\alpha+\tilde{\lambda}, \quad \frac{\partial a}{\partial y}=\frac{\tilde{\mu}}{2}, \quad \frac{\partial b}{\partial x}=\frac{\tilde{\mu}}{2}, \quad \frac{\partial b}{\partial y}=\alpha-\tilde{\lambda} .
$$


By integration of these system, considering $\tilde{\lambda}, \tilde{\mu}$ functions depending only on $z$, we get $a=(\alpha+\tilde{\lambda}) x+\frac{\tilde{\mu}}{2} y+f(z), b=\frac{\tilde{\mu}}{2} x-(\alpha-\tilde{\lambda}) y-g(z), f(z), g(z)$ are arbitrary smooth functions of $z$.

We will continue calculate the tensor fields $\eta, \tilde{\varphi}, \tilde{g}$ and $\tilde{h}$ with respect to the basis $\frac{\partial}{\partial x}, \frac{\partial}{\partial y}, \frac{\partial}{\partial z}$. The expression of the 1-form $\eta=d z$ immediately follows from $\eta(\xi)=1, \eta(X)=\eta(\tilde{\varphi} X)=0$. For the components of $\tilde{g}_{i j}$ of the pseudo-Riemannian metric and the components of the tensor field $\tilde{\varphi}$, the proof is the same with that of Theorem 4.1, we omit here. The components of the tensor field $\tilde{h}$ with respect to the basis $\frac{\partial}{\partial x}, \frac{\partial}{\partial y}, \frac{\partial}{\partial z}$ are given as follows:

$$
\begin{gathered}
\tilde{h}\left(\frac{\partial}{\partial x}\right)=\tilde{h}(X)=\tilde{\lambda} \tilde{\varphi} X=\tilde{\lambda} \frac{\partial}{\partial y}, \tilde{h}\left(\frac{\partial}{\partial y}\right)=\tilde{h} \tilde{\varphi} X=-\tilde{\lambda} X=-\tilde{\lambda} \frac{\partial}{\partial x}, \\
\tilde{h}\left(\frac{\partial}{\partial z}\right)=\tilde{h}\left(\xi-a \frac{\partial}{\partial x}-b \frac{\partial}{\partial y}\right)=b \tilde{\lambda} \frac{\partial}{\partial x}-a \tilde{\lambda} \frac{\partial}{\partial y} .
\end{gathered}
$$

Thus the matrix form of $\tilde{h}$ is given by

$$
\left(\begin{array}{ccc}
0 & \tilde{\lambda} & b \tilde{\lambda} \\
-\tilde{\lambda} & 0 & -a \tilde{\lambda} \\
0 & 0 & 0
\end{array}\right)
$$

Theorem 4.1 and Theorem 4.2 allow us to obtain a complete local classification of 3 -dimensional almost $\alpha$-para-Kenmotsu $(\tilde{\kappa}, \tilde{\mu}, \tilde{\nu}=$ const. $)$-spaces with $\tilde{h}$ is of $\mathfrak{h}_{1}$ type or $\mathfrak{h}_{3}$ type and $d \tilde{\kappa} \wedge \eta=0$. In fact, we can construct in $\mathbb{R}^{3}$ almost $\alpha$-para-Kenmotsu $(\tilde{\kappa}, \tilde{\mu}, \tilde{\nu}=$ const. $)$-space for each of them as follows.

Let $M$ be the open submanifold of $\mathbb{R}^{3}$ defined by $M:=\left\{(x, y, z) \in \mathbb{R}^{3}\right\}$ and

$$
\tilde{\lambda}=c e^{-(2 \alpha+\tilde{\nu}) z}, \tilde{\mu}, f, g: M \rightarrow \mathbb{R}
$$

be four smooth functions of $z$, where $\alpha, c, \tilde{\nu}$ are constant functions. Let us denote again by $x, y, z$ the coordinates induced on $M$ by the standard ones on $\mathbb{R}^{3}$. We consider on $M$

$$
\xi=a \frac{\partial}{\partial x}+b \frac{\partial}{\partial y}+\frac{\partial}{\partial z}, \quad \eta=d z,
$$

the pseudo-Riemannian metric $\tilde{g}$, the tensor fields $\tilde{\varphi}$ and $\tilde{h}$ with respect to the basis $\frac{\partial}{\partial x}, \frac{\partial}{\partial y}, \frac{\partial}{\partial z}$ are given by the relations:

$$
\tilde{g}=\left(\begin{array}{ccc}
-1 & 0 & a \\
0 & 1 & -b \\
a & -b & 1-a^{2}+b^{2}
\end{array}\right), \tilde{\varphi}=\left(\begin{array}{ccc}
0 & 1 & -b \\
1 & 0 & -a \\
0 & 0 & 0
\end{array}\right), \tilde{h}=\left(\begin{array}{ccc}
\tilde{\lambda} & 0 & -a \tilde{\lambda} \\
0 & -\tilde{\lambda} & b \tilde{\lambda} \\
0 & 0 & 0
\end{array}\right)
$$


where $a=\alpha x+\left(\frac{\tilde{\mu}}{2}-\tilde{\lambda}\right) y+f(z), b=\left(\frac{\tilde{\mu}}{2}+\tilde{\lambda}\right) x-\alpha y+g(z), \alpha$ is a constant value. It is easy to check that $(M, \tilde{\varphi}, \xi, \eta, \tilde{g})$ ia an almost paracontact metric manifold. Since $d \eta=0$ and $\Phi=-\frac{1}{2} d x \wedge d y+\frac{b}{2} d x \wedge d z-\frac{a}{2} d y \wedge d z$, thus we get $d \Phi=-\alpha d x \wedge d y \wedge d z=$ $2 \alpha \eta \wedge \Phi$, that is to say, $(M, \tilde{\varphi}, \xi, \eta, \tilde{g})$ ia an almost $\alpha$-para-Kenmotsu manifold and that $\left\{X=\frac{\partial}{\partial x}, \tilde{\varphi} X=\frac{\partial}{\partial y}, \xi\right\}$ makes up a global $\tilde{\varphi}$-basis on $M$. Moreover, by direct computation, we get

$$
[X, \tilde{\varphi} X]=0, \quad[X, \xi]=\alpha X+\left(\tilde{\lambda}+\frac{\tilde{\mu}}{2}\right) \tilde{\varphi} X, \quad[\tilde{\varphi} X, \xi]=\left(\frac{\tilde{\mu}}{2}-\tilde{\lambda}\right) X+\alpha \tilde{\varphi} X .
$$

and

$$
\tilde{h}(X)=\tilde{h}\left(\frac{\partial}{\partial x}\right)=\tilde{\lambda} \frac{\partial}{\partial x}=\tilde{\lambda} X, \tilde{h} \tilde{\varphi} X=\tilde{h}\left(\frac{\partial}{\partial y}\right)=-\tilde{\lambda} \frac{\partial}{\partial y}=-\tilde{\lambda} \tilde{\varphi} X, \tilde{h} \xi=0 .
$$

In this case $\tilde{h}$ is of $\mathfrak{h}_{1}$ type with respect to the $\tilde{\varphi}$-basis $\{X, \tilde{\varphi} X, \xi\}$. By the well-known formula

$$
\begin{aligned}
& 2 \tilde{g}\left(\tilde{\nabla}_{Z} W, T\right) \\
= & Z \tilde{g}(W, T)+W \tilde{g}(T, Z)-T \tilde{g}(Z, W)-\tilde{g}(Z,[W, T])+\tilde{g}(W,[T, Z])+\tilde{g}(T,[Z, W])
\end{aligned}
$$

and by (2.3), we obtain the following identities

$$
\begin{gathered}
\tilde{\nabla}_{X} \xi=\alpha X+\tilde{\lambda} \tilde{\varphi} X, \quad \tilde{\nabla}_{\tilde{\varphi} X} \xi=\alpha \tilde{\varphi} X-\tilde{\lambda} X, \quad \tilde{\nabla}_{\xi} X=-\frac{\tilde{\mu}}{2} \tilde{\varphi} X, \quad \tilde{\nabla}_{\xi} \tilde{\varphi} X=-\frac{\tilde{\mu}}{2} X, \\
\tilde{\nabla}_{X} X=\alpha \xi, \quad \tilde{\nabla}_{\tilde{\varphi} X} \tilde{\varphi} X=-\alpha \xi, \quad \tilde{\nabla}_{\tilde{\varphi} X} X=-\tilde{\lambda} \xi, \tilde{\nabla}_{X} \tilde{\varphi} X=-\tilde{\lambda} \xi .
\end{gathered}
$$

By direct calculation we obtain

$$
\begin{gathered}
\tilde{R}(X, \xi) \xi=\left(\tilde{\lambda}^{2}-\alpha^{2}\right) X+\tilde{\mu} \tilde{h} X+\tilde{\nu} \tilde{\varphi} \tilde{h} X, \\
\tilde{R}(\tilde{\varphi} X, \xi) \xi=\left(\tilde{\lambda}^{2}-\alpha^{2}\right) \tilde{\varphi} X+\tilde{\mu} \tilde{h} \tilde{\varphi} X+\tilde{\nu} \tilde{\varphi} \tilde{h} \tilde{\varphi} X, \\
\tilde{R}(X, \tilde{\varphi} X) \xi=0 .
\end{gathered}
$$

Therefore, for any $Z, W$ on $M$, it holds

$$
\tilde{R}(Z, W) \xi=(\tilde{\kappa} I+\tilde{\mu} \tilde{h}+\tilde{\nu} \tilde{\varphi} \tilde{h})(\eta(W) Z-\eta(Z) W),
$$

and since $\tilde{\kappa}=\tilde{\lambda}^{2}-\alpha^{2}=c^{2} e^{-2(2 \alpha+\tilde{\nu}) z}-\alpha^{2}$, it satisfies $d \tilde{\kappa} \wedge \eta=0$. In this way, we construct an almost $\alpha$-para-Kenmotsu $(\tilde{\kappa}, \tilde{\mu}, \tilde{\nu}=$ const. $)$-space with $\tilde{h}$ is of $\mathfrak{h}_{1}$ type and $d \tilde{\kappa} \wedge \eta=0$.

If we consider on $M$

$$
\xi=a \frac{\partial}{\partial x}+b \frac{\partial}{\partial y}+\frac{\partial}{\partial z}, \quad \eta=d z,
$$


the pseudo-Riemannian metric $\tilde{g}$, the tensor fields $\tilde{\varphi}$ and $\tilde{h}$ with respect to the basis $\frac{\partial}{\partial x}, \frac{\partial}{\partial y}, \frac{\partial}{\partial z}$ are given by the relations:

$\tilde{g}=\left(\begin{array}{ccc}-1 & 0 & a \\ 0 & 1 & -b \\ a & -b & 1-a^{2}+b^{2}\end{array}\right), \tilde{\varphi}=\left(\begin{array}{ccc}0 & 1 & -b \\ 1 & 0 & -a \\ 0 & 0 & 0\end{array}\right), \tilde{h}=\left(\begin{array}{ccc}\tilde{\lambda} & 0 & -a \tilde{\lambda} \\ 0 & -\tilde{\lambda} & b \tilde{\lambda} \\ 0 & 0 & 0\end{array}\right)$.

where $a=(\alpha+\tilde{\lambda}) x+\frac{\tilde{\mu}}{2} y+f(z), b=\frac{\tilde{\mu}}{2} x+(\alpha-\tilde{\lambda}) y+g(z), \alpha$ is a constant value. It is also easy to check that $(M, \tilde{\varphi}, \xi, \eta, \tilde{g})$ is an almost $\alpha$-para-Kenmotsu manifold and that $\left\{X=\frac{\partial}{\partial x}, \tilde{\varphi} X=\frac{\partial}{\partial y}, \xi\right\}$ makes up a global $\tilde{\varphi}$-basis on $M$. Moreover, by direct calculation, we get

$$
[X, \tilde{\varphi} X]=0, \quad[X, \xi]=(\alpha+\tilde{\lambda}) X+\frac{\tilde{\mu}}{2} \tilde{\varphi} X, \quad[\tilde{\varphi} X, \xi]=\frac{\tilde{\mu}}{2} X+(\alpha-\tilde{\lambda}) \tilde{\varphi} X .
$$

and

$$
\tilde{h}(X)=\tilde{h}\left(\frac{\partial}{\partial x}\right)=\tilde{\lambda} \frac{\partial}{\partial y}=\tilde{\lambda} \tilde{\varphi} X, \tilde{h} \tilde{\varphi} X=\tilde{h}\left(\frac{\partial}{\partial y}\right)=-\tilde{\lambda} \frac{\partial}{\partial x}=-\tilde{\lambda} X, \tilde{h} \xi=0 .
$$

In this case $\tilde{h}$ is of $\mathfrak{h}_{3}$ type with respect to the $\tilde{\varphi}$-basis $\{X, \tilde{\varphi} X, \xi\}$.

By the well-known Koszul's formula and by (2.3), we obtain the following identities

$$
\begin{gathered}
\tilde{\nabla}_{X} \xi=(\alpha+\tilde{\lambda}) X, \quad \tilde{\nabla}_{\tilde{\varphi} X} \xi=(\alpha-\tilde{\lambda}) \tilde{\varphi} X, \quad \tilde{\nabla}_{\xi} X=-\frac{\tilde{\mu}}{2} \tilde{\varphi} X, \quad \tilde{\nabla}_{\xi} \tilde{\varphi} X=-\frac{\tilde{\mu}}{2} X, \\
\tilde{\nabla}_{X} X=(\alpha+\tilde{\lambda}) \xi, \quad \tilde{\nabla}_{\tilde{\varphi} X} \tilde{\varphi} X=(\tilde{\lambda}-\alpha) \xi, \quad \tilde{\nabla}_{\tilde{\varphi} X} X=0, \quad \tilde{\nabla}_{X} \tilde{\varphi} X=0 .
\end{gathered}
$$

After long but direct calculation we obtain

$$
\begin{gathered}
\tilde{R}(X, \xi) \xi=-\left(\tilde{\lambda}^{2}+\alpha^{2}\right) X+\tilde{\mu} \tilde{h} X+\tilde{\nu} \tilde{\varphi} \tilde{h} X \\
\tilde{R}(\tilde{\varphi} X, \xi) \xi=-\left(\tilde{\lambda}^{2}+\alpha^{2}\right) \tilde{\varphi} X+\tilde{\mu} \tilde{h} \tilde{\varphi} X+\tilde{\nu} \tilde{\varphi} \tilde{h} \tilde{\varphi} X \\
\tilde{R}(X, \tilde{\varphi} X) \xi=0 .
\end{gathered}
$$

therefore, for any $Z, W$ on $M$, it holds

$$
\tilde{R}(Z, W) \xi=(\tilde{\kappa} I+\tilde{\mu} \tilde{h}+\tilde{\nu} \tilde{\varphi} \tilde{h})(\eta(W) Z-\eta(Z) W),
$$

And since $\tilde{\kappa}=-\left(\tilde{\lambda}^{2}+\alpha^{2}\right)=-c^{2} e^{-2(2 \alpha+\tilde{\nu}) z}-\alpha^{2}$, it satisfies $d \tilde{\kappa} \wedge \eta=0$. In this way, we construct an almost $\alpha$-para-Kenmotsu $(\tilde{\kappa}, \tilde{\mu}, \tilde{\nu}=$ const. $)$-space with $\tilde{h}$ is of $\mathfrak{h}_{3}$ type and $d \tilde{\kappa} \wedge \eta=0$. 


\section{Further Characterizations}

Proposition 5.1. Let $\left(M^{3}, \tilde{\varphi}, \xi, \eta, \tilde{g}\right)$ be an almost $\alpha$-para-Kenmotsu $(\tilde{\kappa}, \tilde{\mu}, \tilde{\nu}=$ const.)-space with $\tilde{h}^{2} \neq 0$ and $d \tilde{\kappa} \wedge \eta=0$. Then the leaves of the canonical foliation of $M$ are flat para-Kähler manifolds.

Proof. Let $M^{\prime}$ be a leaf of $\mathcal{D}$ and $(J,<,>)$ be the induced almost para-Hermitain structure. $M^{\prime}$ is a para-Kähler manifold since it is almost para-Kähler manifold of dimension 2. In order to prove the flatness of $\left(M^{\prime},<,>\right)$, we consider the Weingarten operator $A$ of $M^{\prime}$, if $\tilde{h}$ is of $\mathfrak{h}_{1}$ type, then $A X=-\alpha X-\tilde{\varphi} \tilde{h} X=-(\alpha X+\tilde{\lambda} \tilde{\varphi} X)$ for a unit timelike vector field $X$ such that $\tilde{h} X=\tilde{\lambda} X$ and using the Gauss equation, the sectional curvature $K^{\prime}$ of $<,>$ is given by $K^{\prime}(X, \tilde{\varphi} X)=K(X, \tilde{\varphi} X)-\left(\alpha^{2}+\tilde{\lambda}^{2}\right)$. By Lemma 3.4, we obtain $\tilde{R}(X, \tilde{\varphi} X) \tilde{\varphi} X=-\left(\alpha^{2}+\tilde{\lambda}^{2}\right) X$, thus $K(X, \tilde{\varphi} X)=-\left(\alpha^{2}+\right.$ $\left.\tilde{\lambda}^{2}\right) \tilde{g}(X, X)=\alpha^{2}+\tilde{\lambda}^{2}$. Therefore, we get $K^{\prime}(X, \tilde{\varphi} X)=0$. If $\tilde{h}$ is of $\mathfrak{h}_{3}$ type, then $A X=-\alpha X-\tilde{\varphi} \tilde{h} X=-(\alpha+\tilde{\lambda}) X$ for the unit timelike vector field $X$ such that $\tilde{h} X=\tilde{\lambda} \tilde{\varphi} X$, and using the Gauss equation, the sectional curvature $K^{\prime}$ of $<,>$ is given by $K^{\prime}(X, \tilde{\varphi} X)=K(X, \tilde{\varphi} X)+\tilde{\lambda}^{2}-\alpha^{2}$. By Lemma 3.5, we obtain $K(X, \tilde{\varphi} X)=\tilde{R}(X, \tilde{\varphi} X, \tilde{\varphi} X, X)=\alpha^{2}-\tilde{\lambda}^{2}$. Therefore, we get $K^{\prime}(X, \tilde{\varphi} X)=0$.

Remark 5.1. This conclusion is in accord with Corollary 3 of [9].

Proposition 5.2. Let $\left(M^{3}, \tilde{\varphi}, \xi, \eta, \tilde{g}\right)$ be an almost $\alpha$-para-Kenmotsu $(\tilde{\kappa}, \tilde{\mu}, \tilde{\nu}=$ const.)-space. If $\tilde{h}$ is of $\mathfrak{h}_{1}$ type, then

$$
\mathcal{L}_{\xi} \tilde{h}=-(2 \alpha+\tilde{\nu}) \tilde{h}+\tilde{\mu} \tilde{h} \tilde{\varphi}-2 \tilde{\lambda}^{2} \tilde{\varphi}
$$

If $\tilde{h}$ is of $\mathfrak{h}_{3}$ type, then

$$
\mathcal{L}_{\xi} \tilde{h}=-(2 \alpha+\tilde{\nu}) \tilde{h}+\tilde{\mu} \tilde{h} \tilde{\varphi}+2 \tilde{\lambda}^{2} \tilde{\varphi}
$$

Proof. By (2.3) and (3.12), it is easy to get that

$$
\mathcal{L}_{\xi} \tilde{h}=\tilde{\nabla}_{\xi} \tilde{h}+\tilde{h}(\tilde{\nabla} \xi)-(\tilde{\nabla} \xi) \tilde{h}=-(2 \alpha+\tilde{\nu}) \tilde{h}+\tilde{\mu} \tilde{h} \tilde{\varphi}-2 \tilde{h}^{2} \tilde{\varphi}
$$

Hence, If $\tilde{h}$ is of $\mathfrak{h}_{1}$ type, $\tilde{h}^{2} X=\tilde{\lambda}^{2} X$, If $\tilde{h}$ is of $\mathfrak{h}_{3}$ type, $\tilde{h}^{2} X=-\tilde{\lambda}^{2} X$, the relations (5.1) and (5.2) are easily obtained.

Now we give the following further characterization.

Theorem 5.1. Let $\left(M^{3}, \tilde{\varphi}, \xi, \eta, \tilde{g}\right)$ be an almost paracontact metric manifold $\tilde{h}^{2} \neq$ 0 , and $\tilde{\kappa}, \tilde{\mu}$ are smooth functions on $M$ such that $d \tilde{\kappa} \wedge \eta=0$. Then, $M^{3}$ is an almost $\alpha$-para-Kenmotsu $(\tilde{\kappa}, \tilde{\mu}, \tilde{\nu}=$ const. $)$-space if and only if for any point $p \in M$, there exists an open neighbourhood $U$ of $p$ with coordinates $x_{1}, x_{2}, t$ such that $\tilde{\kappa}$ and $\tilde{\mu}$ 
depend only on $t$ and the tensor fields of the structure are expressed in the following way:

(5.3) $\tilde{\varphi}=\sum_{i, j=1}^{2} \tilde{\varphi}_{j}^{i} d x_{j} \otimes \frac{\partial}{\partial x_{i}}, \xi=\frac{\partial}{\partial t}, \eta=d t, \tilde{g}=d t \otimes d t+\sum_{i, j=1}^{2} \tilde{g}_{i j} d x_{i} \otimes d x_{j}$,

where $\tilde{\varphi}_{j}^{i}, \tilde{g}_{i j}$ are functions only of $t$; The fundamental 2-form $\Phi$ is given by

$$
\Phi=e^{2 t} d x_{1} \wedge d x_{2}
$$

and the non-zero components $\tilde{h}_{j}^{i}, \tilde{B}_{j}^{i}$ in $U$ of $\tilde{h}$ and $B:=\tilde{\varphi} \tilde{h}$, respectively, are functions of $t$ satisfying the condition $\sum_{k} B_{k}^{i} B_{j}^{k}=e^{-2(2 \alpha+\tilde{\nu}) t} \delta_{j}^{i}$ and the following system of differential equations:

$$
\begin{array}{r}
\frac{d \tilde{\varphi}_{j}^{i}}{d t}=2 \tilde{h}_{j}^{i}, \frac{d \tilde{h}_{j}^{i}}{d t}=\mp 2 \tilde{\lambda}^{2} \tilde{\varphi}_{j}^{i}-(2 \alpha+\tilde{\nu}) \tilde{h}_{j}^{i}-\tilde{\mu} \tilde{B}_{j}^{i} \\
\frac{d \tilde{B}_{j}^{i}}{d t}=-(2 \alpha+\tilde{\nu}) \tilde{B}_{j}^{i}-\tilde{\mu} \tilde{h}_{j}^{i}
\end{array}
$$

where $\tilde{\lambda}=e^{-(2 \alpha+\tilde{\nu}) t}$, and it takes " - " if $\tilde{h}$ is of $\mathfrak{h}_{1}$ type, it takes " + if $\tilde{h}$ is of $\mathfrak{h}_{3}$ type.

Proof. Suppose that $M$ carries a structure locally represented as in (5.3)-(5.5). Obviously $d \eta=0$ and $d \Phi=2 \eta \wedge \Phi$ are followed by (5.3)-(5.4), therefore, $M$ is an almost $\alpha$-para-Kenmotsu manifold. Now we need to prove that $M$ satisfies the $(\tilde{\kappa}, \tilde{\mu}, \tilde{\nu}=$ const. $)$-nullity condition. Notice that $X_{1}=\frac{\partial}{\partial x_{1}}$ and $X_{2}=\frac{\partial}{\partial x_{2}}$ are Killing vector fields and thus we get $\tilde{g}\left(\tilde{\nabla}_{X_{i}} X_{j}, X_{k}\right)=0$ for any $i, j, k \in\{1,2\}$. Since the distribution orthogonal to $\xi=\frac{\partial}{\partial t}$ is spanned by $X_{1}$ and $X_{2}$, it follows that $\tilde{\nabla}_{X_{i}} X_{j} \in[\xi]$ for all $i, j \in\{1,2\}$. Consequently, for the Levi-Civita connection $\tilde{\nabla}$ determined by $\tilde{g}$, we obtain

$(5.6) \tilde{\nabla}_{X_{i}} X_{j}=\tilde{\nabla}_{X_{j}} X_{i}=-\tilde{g}\left(X_{i}, \alpha X_{j}+B X_{j}\right) \xi, \quad \tilde{\nabla}_{\xi} X_{i}=\tilde{\nabla}_{X_{i}} \xi=\alpha X_{i}+B X_{i}$.

Using (5.5) and (5.6) and by direct computations, we get

$$
\tilde{R}\left(X_{i}, X_{j}\right) \xi=0,
$$

and

$$
\begin{aligned}
\tilde{R}\left(X_{i}, \xi\right) \xi & =-\tilde{\nabla}_{\xi} \tilde{\nabla}_{X_{i}} \xi=-\alpha\left(\alpha X_{i}+B X_{i}\right)-\left[\frac{d B_{i}^{k}}{d t} X_{k}+B_{i}^{k} \tilde{\nabla}_{\xi} X_{i}\right] \\
& =-\alpha^{2} X_{i}-2 \alpha B X_{i}+(2 \alpha+\tilde{\nu}) B X_{i}+\tilde{\mu} \tilde{h} X_{i}-B^{2} X_{i} \\
& =\left(\tilde{h}^{2}-\alpha^{2} I\right) X_{i}+\tilde{\mu} \tilde{h} X_{i}+\tilde{\nu} \tilde{\varphi} \tilde{h} X_{i} .
\end{aligned}
$$

If $\tilde{h}$ is of $\mathfrak{h}_{1}$ type, $\tilde{R}\left(X_{i}, \xi\right) \xi=\left(\tilde{\lambda}^{2}-\alpha^{2}\right) X_{i}+\tilde{\mu} \tilde{h} X_{i}+\tilde{\nu} \tilde{\varphi} \tilde{h} X_{i}$. Thus, $M^{3}$ is an almost $\alpha$-para-Kenmotsu $(\tilde{\kappa}, \tilde{\mu}, \tilde{\nu}=$ const. $)$-space, where $\tilde{\kappa}=\tilde{\lambda}^{2}-\alpha^{2}$. If $\tilde{h}$ is of $\mathfrak{h}_{3}$ type, 
$\tilde{R}\left(X_{i}, \xi\right) \xi=-\left(\tilde{\lambda}^{2}+\alpha^{2}\right) X_{i}+\tilde{\mu} \tilde{h} X_{i}+\tilde{\nu} \tilde{\varphi} \tilde{h} X_{i}$. Thus, $M^{3}$ is an almost $\alpha$-para-Kenmotsu $(\tilde{\kappa}, \tilde{\mu}, \tilde{\nu}=$ const. $)$-space, where $\tilde{\kappa}=-\left(\tilde{\lambda}^{2}+\alpha^{2}\right)$.

Suppose $M^{3}$ is an almost $\alpha$-para-Kenmotsu $(\tilde{\kappa}, \tilde{\mu}, \tilde{\nu}=$ const. $)$-space, we have (5.3)-(5.5) as similar as the proof of Theorem 6.1 in [16], we omit here.

\section{REF ER E N C ES}

1. B. C. Montano and I. K. Erken: Nullity conditions in paracontact geometry. Diff. Geom. Appl. 30 (2012), 665-693.

2. B. C. Montano and L. D. Terlizzi: Geometric structures associated to a contact metric $(\kappa, \mu)$-space. Pacific J. Math. 246 (2010) 257-292.

3. D. E. Blair: Two remarks on contact metric structures. Tôhoku Math. J. 29 (1977), 319-324.

4. D. E. Blair, T. Koufogiorgos and B. J. Papantoniou: Contact metric manifolds satisfying a nullity condition. Israel J. Math. 91 (1995), no. 1-3, 189C214.

5. G. Dileo and A. M. PAstore: Almost Kenmotsu manifolds and local symmetry . Bull. Belg. math. Soc. Simon Stevin. 14 (2007), 343-354.

6. G. Dileo and A. M. Pastore: Almost Kenmotsu manifolds and nullity distributions. J.Geom. 93 (2009), 46-61.

7. G. DiLeo: On the geometry of almost contact metric manifolds of Kenmotsu type . Differential Geom. Appl. 29(2011) 558-564.

8. H. Öztürk, N. Aktan rm and C. Murathan: Almost $\alpha$-cosymplectic $(\kappa, \mu, \nu)$-spaces. arXiv:1007.0527v1[math.DG]

9. I. K. Erken, P. Dacko and C. Murathan: Almost a-paracosymplectic manifolds. J. Geom. Phys. 88 (2015), 30-51.

10. K. Kenmotsu: A class of almost contact Riemannian manifolds. Tôhoku Math. J. 24 (1972), 93-103.

11. T. Koufogiongos and C. Tsichlias: On the existence of a new class of contact metric manifolds. Canadian Math. Bull. 43(2000), 440-447.

12. P. DACKo and Z. Olszak: On almost cosymplectic $(\kappa, \mu, \nu)$-spaces. Banach Center Publ., 69 2005, 211-220.

13. P. DАско: On almost para-cosymplectic manifolds. Tsukuba J. Math. 28 (2004), 193-213.

14. S. Kaneyuki RM AND F. Williams: Almost paracontact and parahodge structures on manifolds. Nagoya Math. J. 99(1985), 173-187.

15. S. Zamkovoy: Canonical connections on paracontact manifolds. Ann.Glob. Anal. Geom. 36(2009), 37-60.

16. V. SAltaRelLI: Three-dimensional almost Kenmotsu manifolds satisfying certain nullity conditions . Bull. Malays. Math. Sci. Soc. 38(2015), 437-459. 
Ximin Liu

School of Mathematical Sciences Dalian Universityof Technology

Dalian 116024, China

ximinliu@dlut.edu.cn

Quanxiang Pan

School of Mathematical Sciences

Dalian Universityof Technology

Dalian 116024, China

panquanxiang@dlut.edu.cn 